Supporting Information

\title{
Electrically Adaptive and Shape-Changeable Invertible Microlens
}

Jin Woo Bae ${ }^{\dagger}$, Dong-Soo Choi ${ }^{\dagger}$, In-Ho Yun, Dong-Heon Han, Seung-Ju Oh, Tae-Hoon Kim, Jeong Ho Cho, Liwei Lin", Sang-Youn Kim*

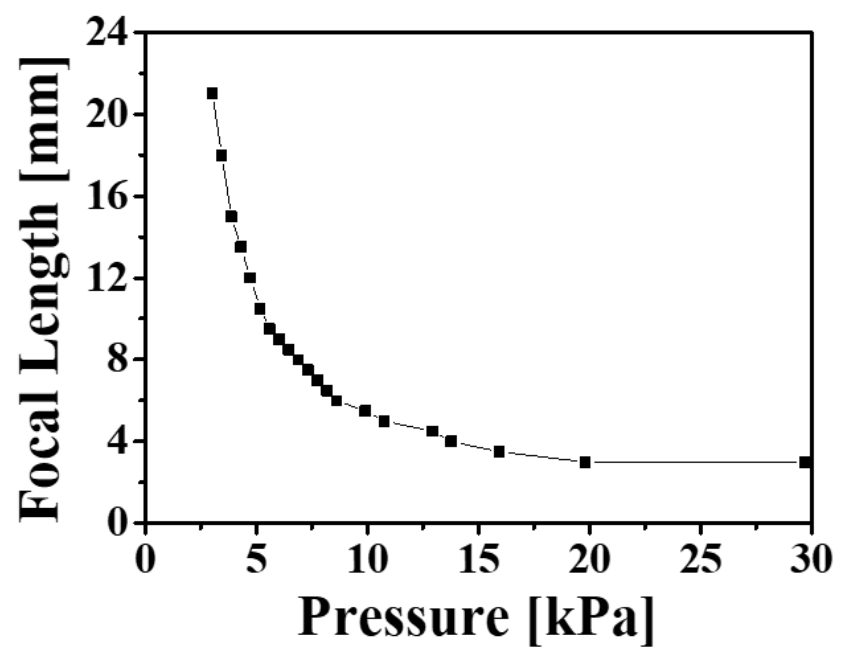

Figure S1. Initial focal length variation as a function of compression pressure.

\section{Description of Figure $S 1$}

The SPNIPGel was sandwiched between two plates with the annular electrode and then squeezed together under a specified pressure. The pressure caused the SPNIPGel to bulge outward into the small hole, resulting in a change in the initial focal length of the proposed lens. Figure S1 shows the initial focal length of the proposed lens as a function of the applied pressure.

a

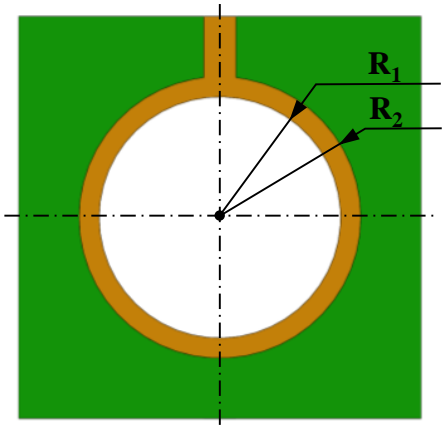

$\mathbf{b}$

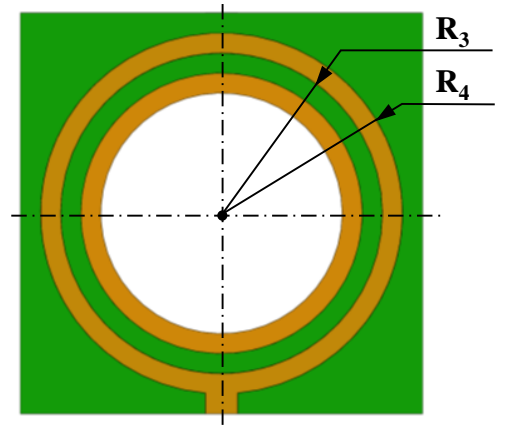

Figure S2. Plate structure of two annular electrodes (anode and cathode). The two annular electrodes (anode and cathode) were at a 0.2-mm distance from each other: a) the anode electrode had inner and outer radii of $R_{1}=0.75 \mathrm{~mm}$ and $R_{2}=0.9 \mathrm{~mm}$, respectively, and $b$ ) the cathode had inner and outer radii of $R_{3}=1.1 \mathrm{~mm}$ and $R_{4}=1.5 \mathrm{~mm}$, respectively. 
$\mathbf{a}$

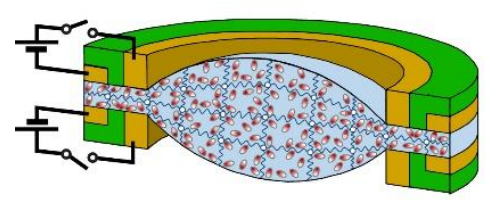

: DBA plasticizer

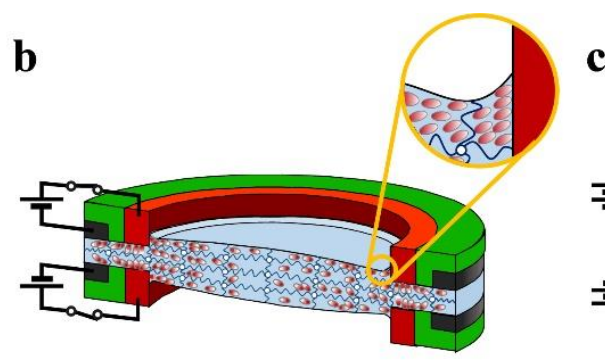

c

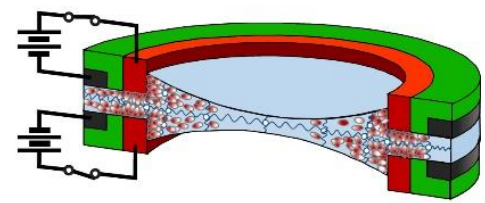

W : Polymer chain

$\bigcirc$ : Physical cross-linking point

Figure S3. Operating principle of the proposed microlens based on SPNIPGel. (a) Unactivated state without electric field. (b) the activated state with low electric field. (c) the activated state with high electric field.

\section{Description of Figure S3}

The operating principle of the proposed electrically tunable microlens is attributed to the electric-field-induced creep deformation of the SPNIPGel resulting from the applied voltage ${ }^{8,16,17}$ Figure S3a shows the initial state of the proposed lens based on the SPNIPGel under no electric field. When a voltage is applied to the SPNIPGel, the dipoles in the $\mathrm{PVC} / \mathrm{P}(\mathrm{VC}-\mathrm{VA})$ chains and DBA plasticizers move to the anode, resulting in the dragging SPNIPGel. This causes an alternation in the initial curvature of the double-convex SPNIPGel surface because of the rubber-like elastic SPNIPGel characteristics maintaining a constant volume (Figure S3b). Because the dipole movement is dependent on the applied electric field, the dipoles of the SPNIPGel under a much higher electric field easily move to the anode; some of them climb up the annular Cu electrode (anode) (Figure S3c). This phenomenon contributes to the surface shapes of the concave SPNIPGel (Figure S3c).
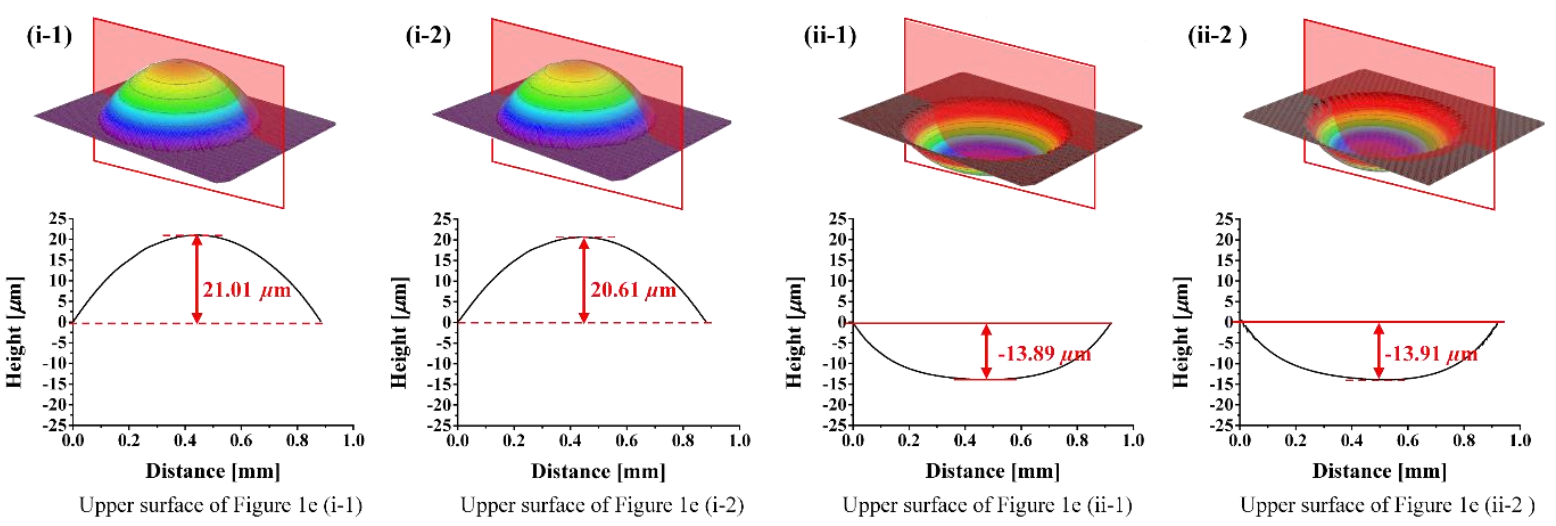

Figure S4. Height of the upper side of the proposed microlens: (i-1) no applied voltage, (i-2) applied voltage to the set of lower electrodes, (ii-1) applied voltage to only the set of upper electrodes, (ii-2) applied voltage to two sets of lower and upper electrodes.

\section{Description of Figure S4}

We could see the peripheral zone of the PVC gel creeps along the anode after voltage ON (Figure S3b). For this reason, we set the effective diameter of the SPNIPGel-based lens as 1 $\mathrm{mm}$ even though the actual diameter of the proposed microlens is $1.5 \mathrm{~mm}$. 


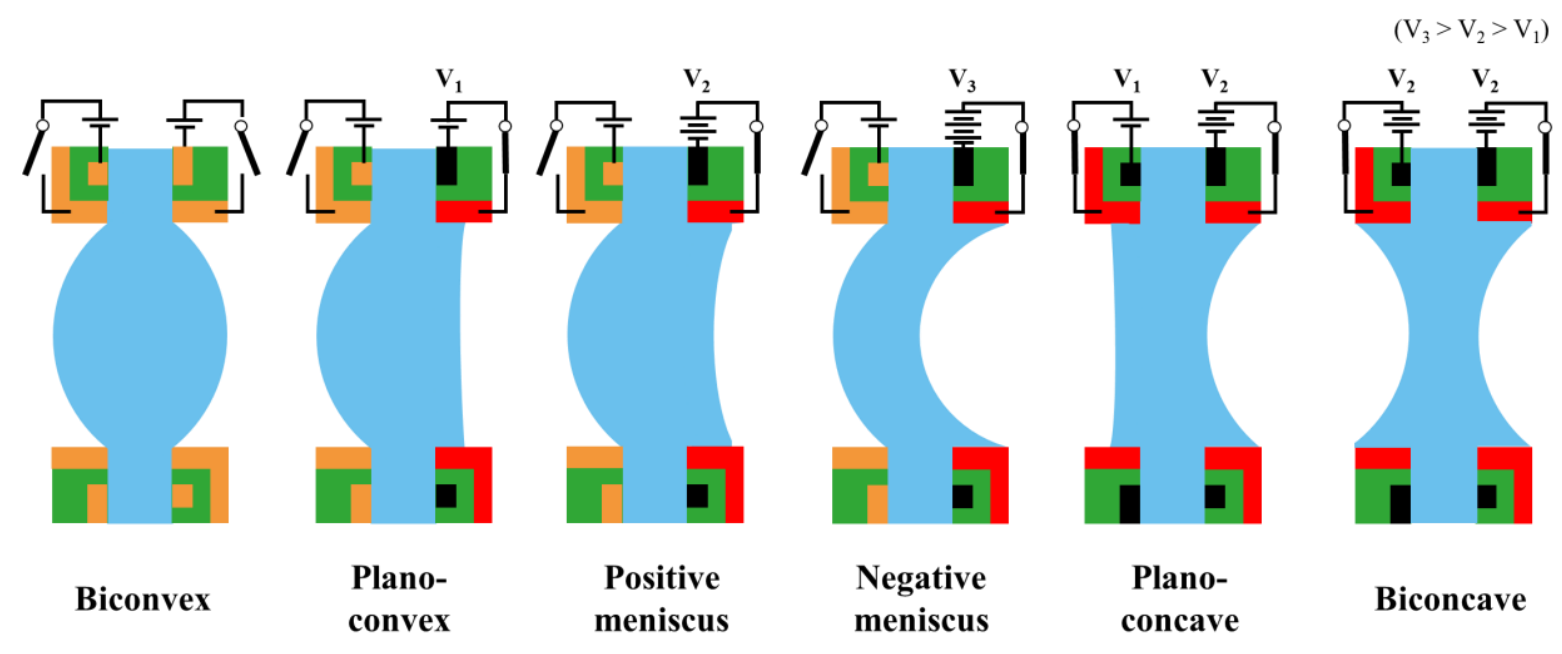

Figure S5. Reconfigurable lens shapes of proposed microlens

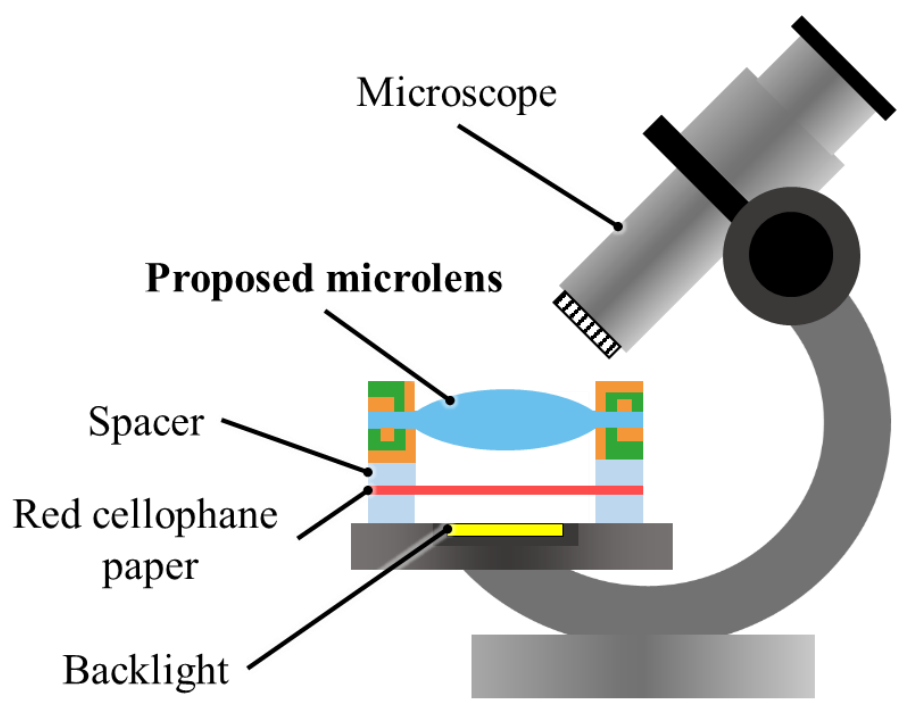

Figure S6. Experimental setup for capturing a picture of the upper surface of the proposed lens.

\section{Description of Figure S6}

It is difficult to capture a picture of the transparent proposed lens. Therefore, red light from the backlight and a red cellophane paper was shed on the lens. Subsequently, we captured a picture of the lens's upper surface by changing the applied electric field. 
a

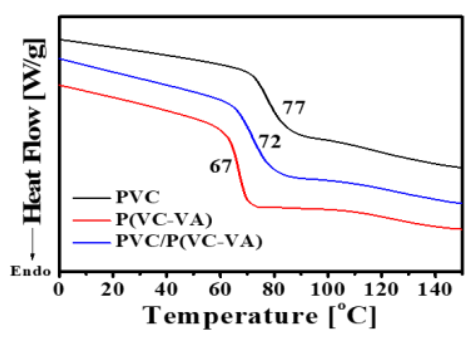

b

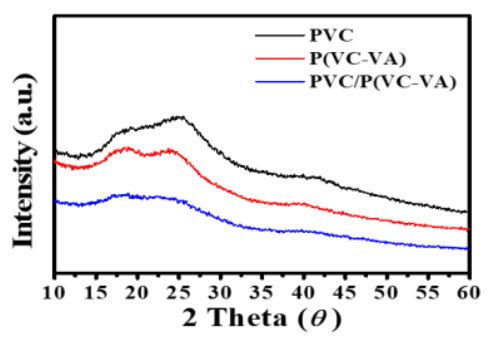

c

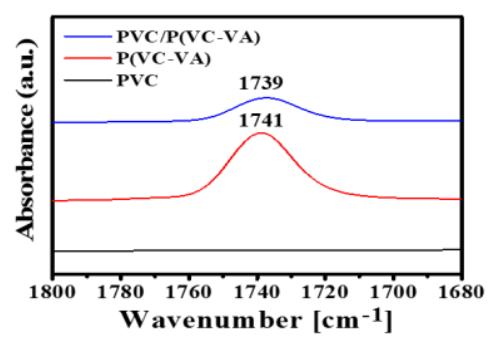

Figure S7. Characteristics of PVC, P(VC-VA), and PVC/P(VC-VA). (a) DSC curves (b) WAXD, and (c) FT-IR of PVC, P(VC-VA), and PVC/P(VC-VA) blend at a 1:1 ratio. In the DSC curves, from the Fox equation $\left(\frac{1}{T_{g}}=\frac{w_{1}}{T_{g 1}}+\frac{w_{2}}{T_{g 2}}\right)$, the calculated $T_{g}$ of the PVC/P(VC-VA) blend film was $72{ }^{\circ} \mathrm{C}$, where $w_{1}$ and $w_{2}$ are the weight fractions of monomers 1 and 2, respectively, and $T_{g 1}$ and $T_{g 2}$ are the $T_{g}$ of Polymers 1 and 2, respectively.

$\mathbf{a}$

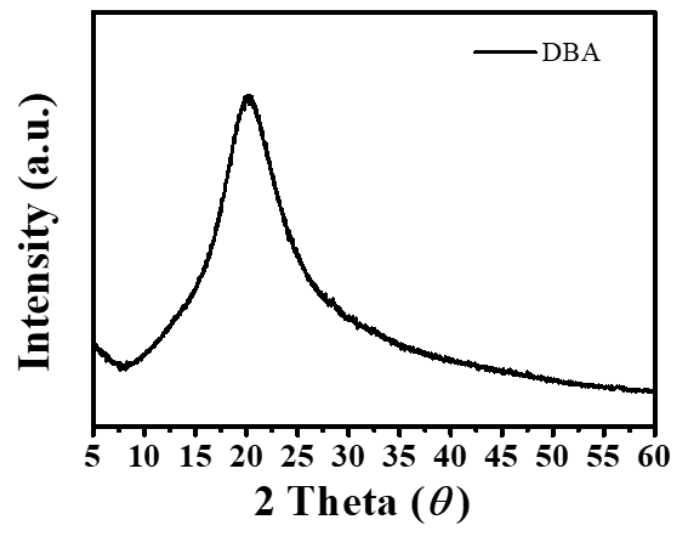

c

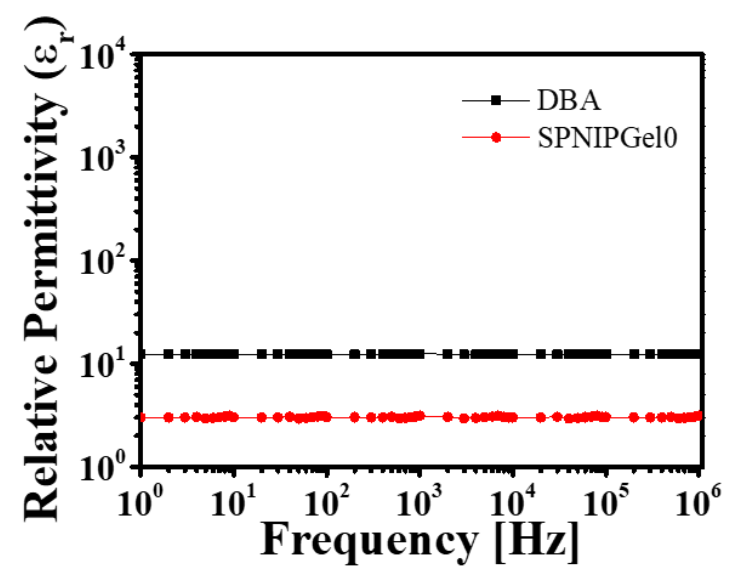

b

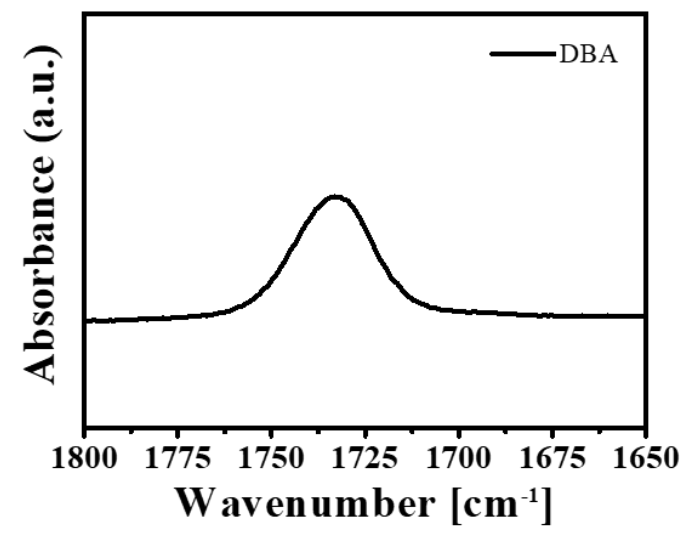

d

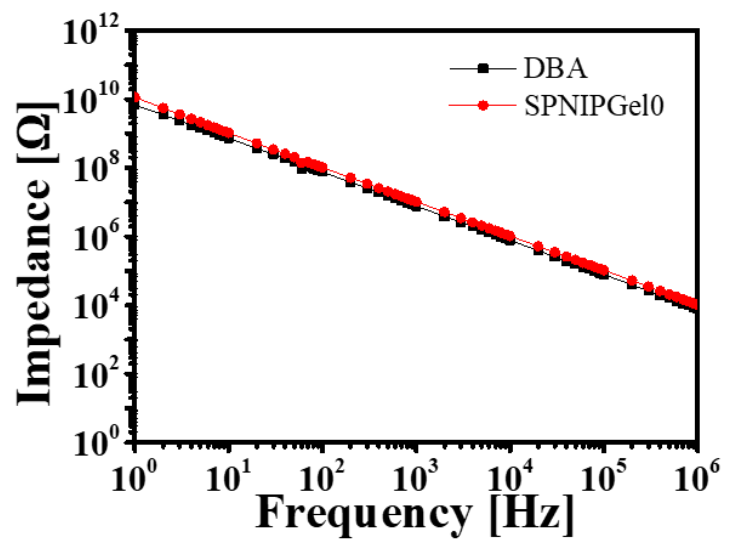

Figure S8. Characteristics of DBA and SPNIPGel0. (a) XRD pattern and (b) FT-IR spectrum of the DBA plasticizer, (c) relative permittivity, and (d) complex impedance of SPNIPGel0 and the DBA plasticizer. 


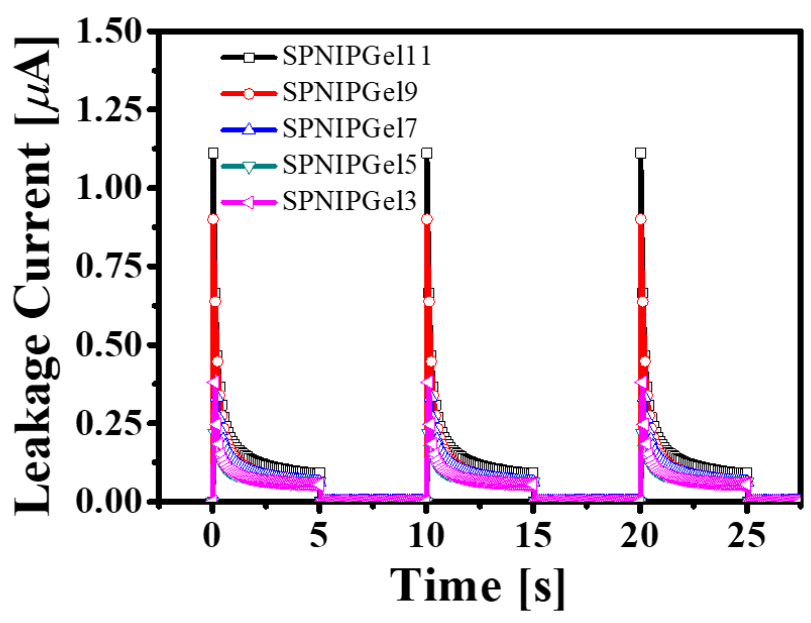

Figure S9. Leakage current of the SPNIPGel under an applied electric field of $400 \mathrm{~V} / \mathrm{mm}$.

$\mathbf{a}$

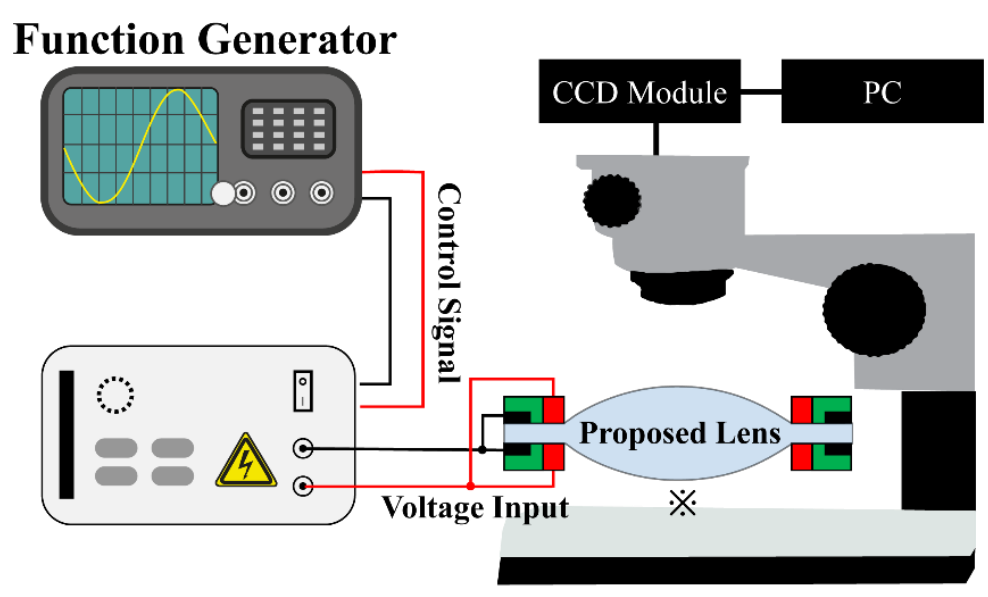

b

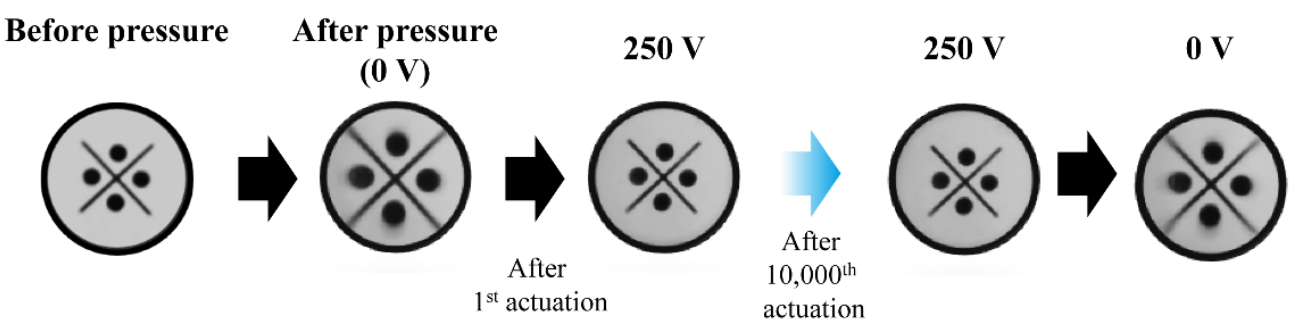

Figure S10. Long-term stability of SPNIPGel-based microlenses (a) the experimental environment to check the repeatability of the proposed SPNIPGel-based microlenses (b) target image observed from the microscope when input voltage of $250 \mathrm{~V}$ (on state) and $0 \mathrm{~V}$ (off state) was alternatively applied, after 1 and 10,000-actuation cycles of the proposed SPNIPGel7-based microlenses. To demonstrate the long-term stability of electro-reconfigurable SPNIPGel-based microlens, a repetitive pulse voltage input of $250 \mathrm{~V}$ and $0 \mathrm{~V}$ was applied. The $250 \mathrm{~V}$ was applied to the microlens during $5 \mathrm{~s}$, and then $0 \mathrm{~V}$ was provided to the microlens for $5 \mathrm{~s}$. Then, this procedure was repeated 10,000 times. 


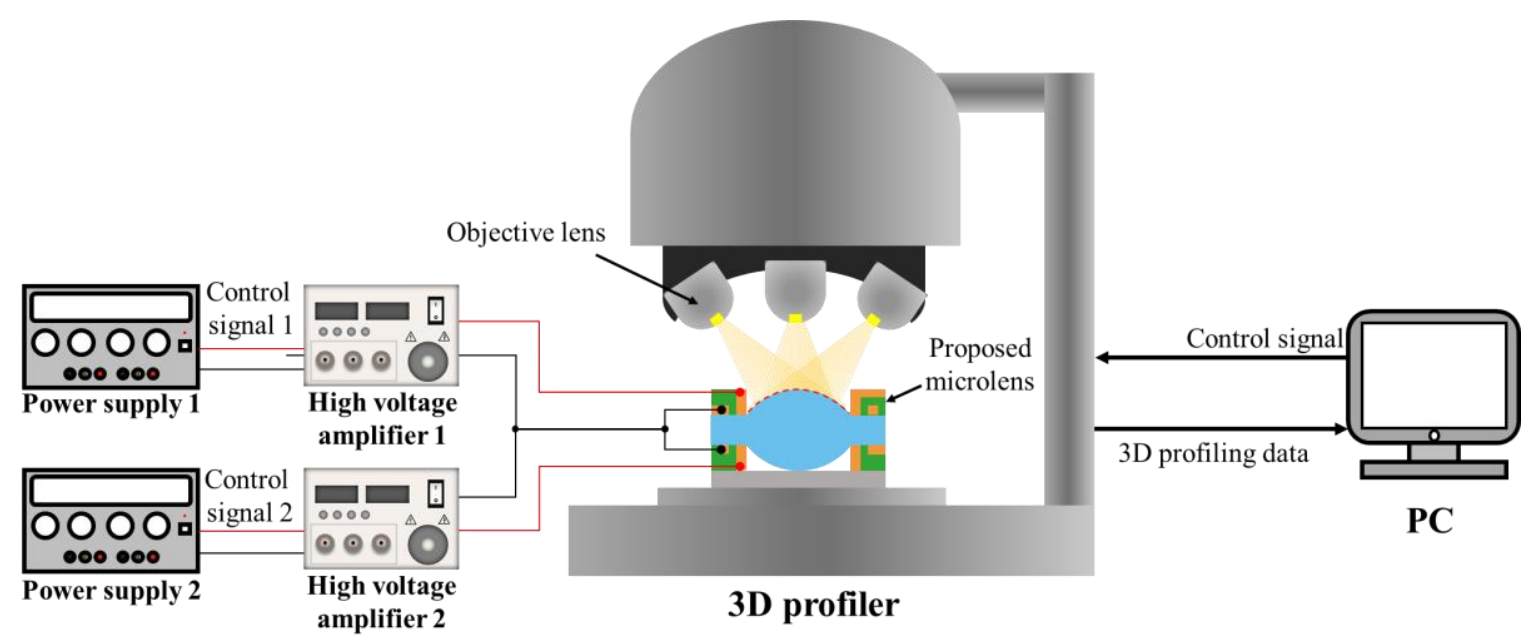

Figure S11. Experimental environment for observing the curvature of proposed lens.

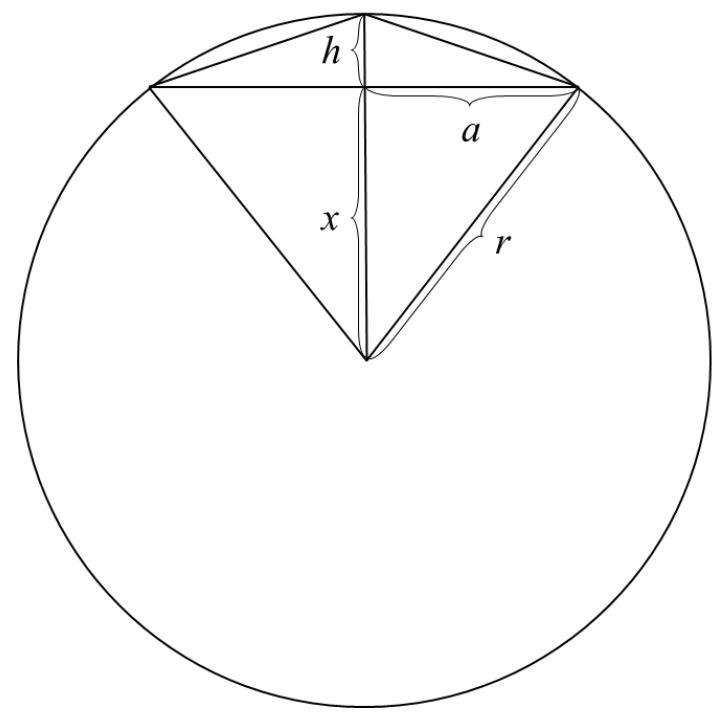

$$
\begin{aligned}
& r=x+h \\
\Rightarrow & r^{2}=a^{2}+x^{2} \\
\Rightarrow & (x+h)^{2}=a^{2}+x^{2} \\
\Rightarrow & x^{2}+2 x h+h^{2}=a^{2}+x^{2} \\
\Rightarrow & 2 x h=a^{2}-h^{2} \\
\Rightarrow & x=\frac{a^{2}-h^{2}}{2 h} \\
\Rightarrow & r=\frac{a^{2}-h^{2}}{2 h}+\mathrm{h}
\end{aligned}
$$

Figure S12. Equation for calculating the curvature radius. 